\title{
Correlation between Uric Acid and Non-Alcoholic Steatohepatitis (NASH) Occurred in Obesity and Non-Obesity
}

\author{
Rasdiana ${ }^{1}$, Faridin $\mathrm{HP}^{1}$, Fardah Akil ${ }^{1}$, AM Luthfi Parewangi ${ }^{1}$, Hasyim Kasim ${ }^{1}$, Himawan Sanusi ${ }^{1}$, Femi Syahriani ${ }^{1}$, \\ Syakib Bakri ${ }^{1}$, Haerani Rasyid ${ }^{1} \&$ Arifin Seweng ${ }^{2}$ \\ ${ }^{1}$ Department of Internal Medicine, Medical Faculty, Hasanuddin University, Makassar, Indonesia \\ ${ }^{2}$ Department of Biostatistics, Public Health Faculty, Hasanuddin University, Makassar, Indonesia \\ Correspondence: Rasdiana, Department of Internal Medicine, Hasanuddin University's Faculty of Medicine, \\ Perintis Kemerdekaan Km 7, Tamalanrea, Makassar, South Sulawesi, Indonesia.
}

Received: October 12, 2020 Accepted: December 1, 2020 Online Published: December 17, 2020

doi:10.5539/gjhs.v13n1p103 URL: https://doi.org/10.5539/gjhs.v13n1p103

\begin{abstract}
Background/Aim: Non-alcoholic Fatty Liver Disease (NAFLD) is one of the main causes that promote chronic liver disease in developing countries. Uric acid is correlated to metabolic syndrome. Based on this issue, we studied the correlation between uric acid level and the occurrence of NASH in non-alcoholic fatty liver disease (NAFLD) with or without obesity.

Methods: The research subjects were 149 patients diagnosed with NAFLD. The data were collected from the medical record by purposive sampling method. The subjects were taken from inpatient and outpatient data from Wahidin Sudirohusodo hospital. The medical record included demography, clinical, radiology and laboratory records. Statistical analysis were performed through descriptive statistical calculations, Pearson Correlation and multinomial logistic.
\end{abstract}

Results: There was a significant correlation between NAFLD and uric acid level $(\mathrm{p}=.000)$. Based on gender, the correlation between NAFLD and uricemia was significant in female patients (with $\mathrm{p}=.000$ ); but insignificant in male patients $(\mathrm{p}=.137)$. Based on age, in age of $>40$ years old, NAFLD was significantly associated with uric acid level $(\mathrm{p}=.000)$. There was a significant correlation between hyperuricemia and NASH in obese and non-obese patients $(\mathrm{p}<0.001)$ for which the higher the uric acid level the greater the NAFLD degree was.

Conclusion: There is a correlation between uric acid level and NASH occurrence in NAFLD with or without obesity.

Keywords: uric acid, Non-Alcoholic Steatohepatitis, obesity, Non-Alcoholic Fatty Liver Disease, Body Mass Index, uricemia

\section{Introduction}

Non-alcoholic Fatty Liver Disease (NAFLD) is one of the main causes of chronic liver disease in developing countries (Lefere et al., 2019; Younossi et al., 2018). The highest prevalence of NAFLD in western countries is found in obese individuals ranging from 80\% -90\% (Bellentani et al., 2010). Obesity [Body Mass Index (BMI) < 25] is associated with a spectrum of liver disorders, known as NAFLD, indicated by an increase in intrahepatic triglycerides so-called simple steatohepatitis (non-NASH) (without inflammation and fibrosis), or Non-Alcoholic Steatohepatitis (NASH) with or without inflammation and fibrosis, which can progress to cirrhosis (Fabbrini et al., 2010). Many studies have reported a relationship between NAFLD and metabolic syndrome (Souza de et al., 2012). Likewise, the correlation between NAFLD and uric acid is researched as well.

Serum uric acid (SUA) is the final outcome of purine metabolism in the human body, and it is derived from hypoxanthine after the double enzyme catalysis, which is performed by xanthine, oxidized in the liver. It is a metabolic product of endogenous (nucleoproteins originating from cellular metabolism) and an exogenous (dietary) precursor protein transferred to the liver, and is excreted by the kidneys. Elevated uric acid levels are able to induce the triglyceride accumulation by promoting the over-expression of pro-lipogenic enzymes so-called sterol regulatory element-binding proteins (Yang et al., 2018). Therefore, any impairment in this balance may lead to high level of SUA (Yang et al., 2017). 
The increasing level of uric acid serum is closely related to visceral fat accumulation, as well as to the onset of hepatic histological that changes independently. In adipocytes, soluble uric acid (UA) stimulates an elevation in reactive oxygen species (ROS) production which has been recently recognized as a major factor that determines the obesity-related endocrine imbalance inflammatory. Therefore, as a high-risk factor, uric acid levels may develop as one of the predictive markers for the incidence and severity of NAFLD, implying that uric acid levels may be a potential therapeutic target for NAFLD, especially in patients with hyperuricemia (Zhang et al., 2018).

Uric acid is correlated to metabolic syndrome (Ali et al., 2020). Hence, this research was conducted to identify the correlation of uric acid levels and NASH in NAFLD (Non-Alcoholic Fatty Liver Disease) with and without obesity.

\section{Method}

\subsection{Sampling Procedures}

This research was an observational study with a cross-sectional approach applied on 149 NAFLD patients. The samples were obtained from medical record data through purposive sampling. The study population was the entire medical records of patients diagnosed with NAFLD, treated outpatient, and inpatient at Wahidin Sudirohusodo Hospital and its Network from 2018 to 2019.

\subsection{Inclusion and Exclusion Criteria}

The inclusion criteria included the patients at $>18$ years in age, no history of consuming alcohol nor alcohol intake in amounts deemed harmful to the liver (less than 20 grams of ethanol per week), while the exclusion criteria were the people with coronary heart, hepatitis A, hepatitis B, hepatitis C, and malignant diseases. The criteria for Non-Alcoholic Fatty Liver Disease were determined based on the image of fatty liver from abdominal ultrasound/CT-scan, Magnetic Resonance Spectroscopy (1H-MRS) or Magnetic Resonance Imaging (MRI).

The obese criteria were determined based on body mass index which was calculated based on body's weight (in kilograms) and height (in meters). The subjects were classified into normal weight (BMI, 18.5-22.9), overweight (BMI, 23-24, 9), Obesity I (BMI, 25-29,9), Obesity II (BMI,> 30). The uric acid levels were considered increased if the value was $>7 \mathrm{mg} / \mathrm{dL}$ for men and $>6 \mathrm{mg} / \mathrm{dL}$ for women.

\subsection{Statistical Analysis}

Data analysis was carried out by using SPSS version 22. Statistical analysis was performed through descriptive statistical calculations, Pearson Correlation and multinomial logistic. The test result would be considered significant if the p-value was $<0.05$. The results were displayed descriptively with tables, narration, and figures.

\subsection{Ethical License}

Ethical permission has been obtained from the Health Research Ethics of the Hasanuddin University Medical Faculty/ Wahidin Sudirohusodo Hospital.

\section{Results and Discussion}

A total of 149 subjects were diagnosed with Non-Alcoholic Fatty Liver Disease (aged $\geq 18$ years).

\subsection{Correlation Analysis: Between NAFLD and Gender, Uric Acid Levels and Age}

Among the 149 subjects, 70 were male and 79 were female. The correlation between non-alcoholic fatty liver and gender was proven statistically. The Pearson correlation was significant $(p=.000)$, indicating that men and women had a different likelihood in experiencing different stages of NAFLD (see Table 1). No correlation was found between NAFLD and age even though we used the same statistics. We divided the ages by the threshold of 40 years old and found no differences between both age groups, see Table 1.

The stage of NAFLD was highly correlated with uric acid levels. We used Pearson's chi-square correlation for the categorical variables. By using all of the samples, we found a significant relationship between NAFLD and patient's uric acid levels $(\mathrm{p}=.000)$, see Table 1 . 
Table 1. Correlation between NAFLD and Patient's Gender, Uric Acid Levels, and Age

\begin{tabular}{|c|c|c|c|}
\hline \multirow{2}{*}{ Variable } & \multicolumn{2}{|c|}{ NAFLD } & \multirow{2}{*}{ Tota } \\
\hline & NASH & Non-NASH & \\
\hline \multicolumn{4}{|l|}{ Gender } \\
\hline Male & 12 & 58 & 70 \\
\hline Female & 43 & 36 & 79 \\
\hline Total & 55 & 94 & 149 \\
\hline \multicolumn{4}{|l|}{ Age } \\
\hline$<40$ years old & 10 & 12 & 22 \\
\hline$>40$ years old & 45 & 82 & 127 \\
\hline Total & 55 & 94 & 149 \\
\hline Normourisemia & 11 & 63 & 74 \\
\hline Hyperuricemia & 44 & 31 & 75 \\
\hline Total & 55 & 94 & 149 \\
\hline
\end{tabular}

Based on Table 1. We found that 45 NASH subjects were aged $>40$ years old and the 10 subjects were aged $<40$ years old. On other hand, we found that the number of non-NASH patients, with the age of $>40$ years old, were 82, and it was 12 for the patients with the age of $<40$ years old. There are 44 subjects suffering from NASH and hyperuricemia. Meanwhile, the other 11 subjects who were suffering from NASH also had normouricemia.

\subsection{Correlation between NAFLD Degree and Uric Acid Levels to Obesity}

Table 2 shows a significant correlation between NASH and hyperuricemia in obese and non-obese patients. The Pearson correlation $(\rho)$ represents the correlation between NAFLD and uricemia for each obese group.

Table 2. Correlation between NASH and non- NASH patients and the Uric Acid Levels in Obese and Non-obese Patients

\begin{tabular}{|c|c|c|c|c|c|c|c|}
\hline & & \multicolumn{4}{|c|}{ Uricemia } & \multirow{3}{*}{$\begin{array}{l}\text { Pearson } \\
\text { Correlation } \\
(\rho)\end{array}$} & \multirow{3}{*}{ Prob. } \\
\hline & & \multicolumn{2}{|c|}{ Hyperuricemia } & \multicolumn{2}{|c|}{ Normouricemia } & & \\
\hline & & $\mathrm{n}$ & $(\%)$ & $\mathrm{n}$ & $(\%)$ & & \\
\hline \multirow{2}{*}{ Obese } & NASH & 29 & $(19.5)$ & 9 & $(6.0)$ & -0.41 & 0.000 \\
\hline & Non- NASH & 22 & $(14.8)$ & 43 & (28.9) & & \\
\hline \multirow{2}{*}{ Non-Obese } & NASH & 15 & $(10.1)$ & 2 & (1.3) & -0.55 & 0.000 \\
\hline & Non- NASH & 9 & $(6.0)$ & 20 & (13.4) & & \\
\hline
\end{tabular}




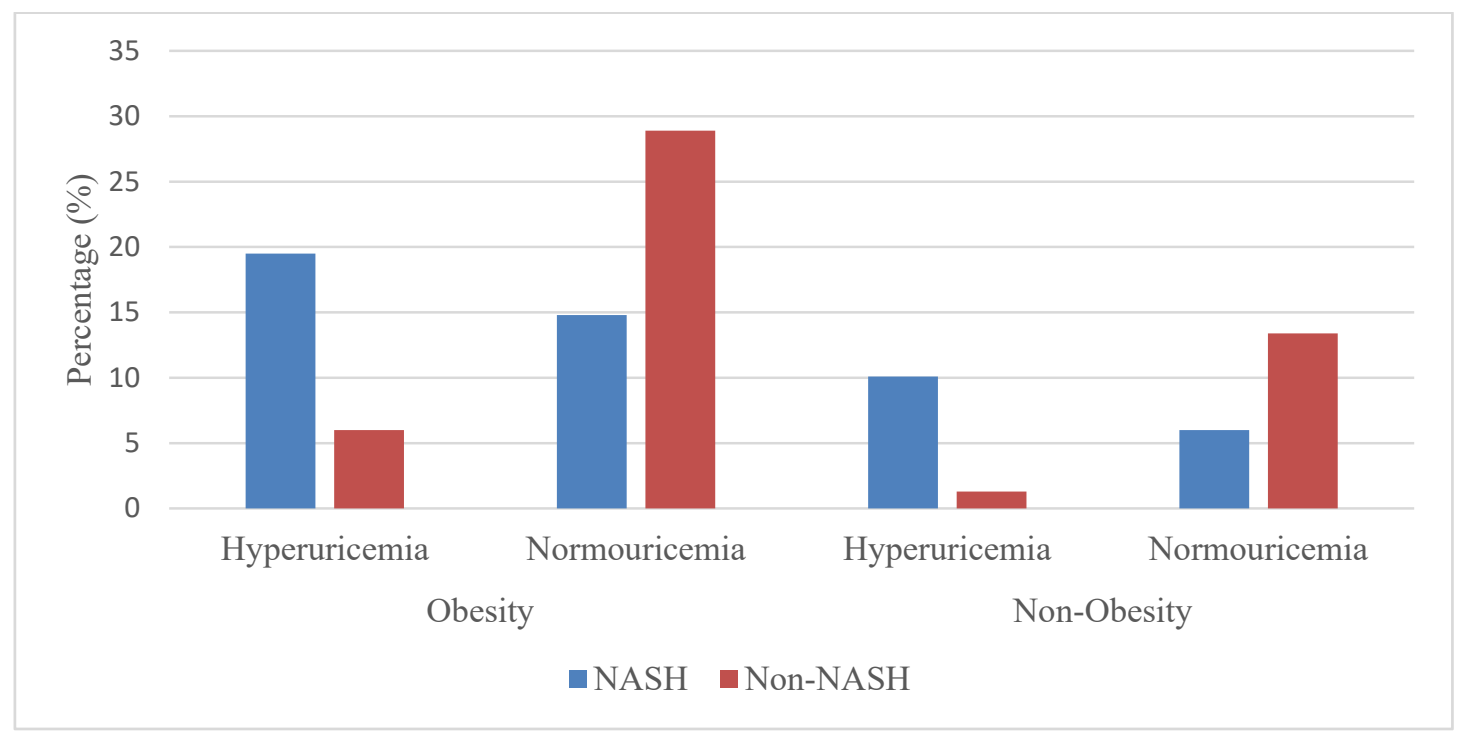

Figure 1. NAFLD Cases based on Uric Acid Levels and Obesity Status

As shown in Table 2, the number of obese patients with NASH and hyperuricemia (19.5\%) was found significantly higher than that of the obese patient group with non-NASH and hyperuricemia (14.8\%). The group of non-obese patients with NASH and hyperuricemia $(10.1 \%)$ had higher rate than the non-obese who suffered from non-NASH and hyperuricemia group (6\%).

Obese patients with NASH and normouricemia (6\%) had lower rate than the obese group with non-NASH and normouricemia (28.9\%). Non-obese patients with NASH and normouricemia group (1.3\%) had lower rate than non-obese group with non-NASH and normouricemia (13.4\%).

This study found a significant correlation between NAFLD and uric acid levels in female patients. Previous studies found a disparity between the two genders about the association between serum uric acid and NAFLD (stronger association was found in women rather than in men) (Jaruvongvanich et al., 2017). This result is in accordance with the study of Yang et al., stating that the independent effect of hyperuricemia on NAFLD was higher in women than in men (Yang et al., 2017). Gender difference concerning the relation between uric acid levels and NAFLD might lay in the fact that estrogen serves as a uricosuric agent, which can lower the uric acid levels through uric degradation and excretion. Some studies also suggest that insulin resistance may not only increase the uric acid synthesis but also decrease the uric acid excretion (Yu et al., 2017). The uricosuric action of estrogen has been suggested to be the mechanism which underlies the gender difference regarding SUA levels or the prevalence of gout (Park et al., 2018).

A study by Ballestri et al. revealed that increased liver injury and inflammation in premenopausal women generates a lower risk of liver fibrosis compared to men and women with the postmenopausal cases. The prevalence of NAFLD is globally higher in men than women, but, after menopause, women display a similar or even a higher NAFLD prevalence compared to men. The findings supported that it is related to the protective effect of estrogens. In some cross-sectional studies, it is stated that male gender and menopausal status have often been connected with the risk of NAFLD aside from the age and metabolic factors. The prevalence of NASH and advanced fibrosis were found to be higher in postmenopausal women than in men. However, other longitudinal studies were unsuccessful to support the role of gender in the progression of liver fibrosis (Ballestri et al., 2017).

Significant risk factors of the reported NAFLD will be obesity, diabetes mellitus (DM) and hypertriglyceridemia (Wong et al., 2019). Obesity, especially central obesity, is associated with insulin resistance through an inflammatory process (Hardy et al., 2012). According to the pathomechanism of NAFLD, based on the theory of "Two Hit", "the first hit" is the occurrence of hepatitis steatosis in which there is an imbalance between the formation and overhaul of triglycerides (Fang et al., 2018). The existence of insulin resistance may have a major influence on the onset of NAFLD (Benedict et al., 2017).

The visceral fat component is metabolically active and regulates numerous adipocytokines, such as leptin and adiponectin, which have been associated with insulin resistance. Insulin resistance or hyperinsulinemia elevates the reabsorption of sodium and uric acid on the renal tubules, thereby decreasing the urinary excretion of uric acid, 
and it may cause hyperuricemia (Kim et al., 2012; Fabbrini et al., 2010).

Uric acid promotes an escalation of the mRNA expression of monocyte chemotactic protein-1 (MCP-1) and decreases the mRNA expression of adiponectin. A strong positive association between serum leptin and UA has been demonstrated in both diabetic and healthy subjects (Zhang et al., 2018). In the patients with non-alcoholic fatty liver disease (NAFLD), hyperuricemia is related to a more severe degree of liver damage (Jaruvongvanich et al., 2017; Yu et al., 2017). High uric acid levels can lead to endothelial dysfunction, insulin resistance, oxidative stress, and systemic inflammation (Wang et al., 2018; Ali et al., 2020). All these factors are involved in NAFLD pathogenesis (Petta et al., 2011). Therefore, high uric acid levels are the cause of the progression from non-NASH to NASH (Jaruvongvanich et al., 2017). Another predictive factor of NAFLD, uric acid, was reportedly responsible for lipid metabolism impairment, including mitochondrial oxidative stress, sterol regulatory element-binding protein 1 (SREBP-1) activation which is induced by endoplasmic reticulum (ER) stress, and involvement of NLRP3 inflammation. Thus, there may be an interaction between high SUA levels and obesity in the pathogenesis of NAFLD (Yang et al., 2017). In addition, some studies have also revealed that insulin resistance may not only increase the uric acid synthesis but also decrease the uric acid excretion (Yu et al., 2017).

This study shows a significant correlation between NASH and hyperuricemia in obese and non-obese patients. The higher the uric acid level, the heavier the degree of NAFLD was. The details are shown in Table 2 and Figure 1. This study found a relationship between UA serum levels and clinically diagnosed NAFLD (ultrasound evidence or laboratory surrogates of steatosis).

This result is consistent with the study conducted by Petta et al. (a cohort study about patients with histological diagnosis of NAFLD) which shows an independent relationship between hyperuricemia and the severity of liver damage, implying that hyperuricemia was independently associated with lobular inflammation. These results indicate that hyperuricemia has an important role in inducing inflammation (Petta et al., 2011). Likewise, the study of Jaruvongvanich et al. shows that patients with NAFLD and hyperuricemia will be more commonly possessing a high NAS (defined as score of $\geq 5$ ), in a significant way, rather than those without hyperuricemia with a pooled odds ratio of 2.17 (95\% confidence interval: 1.51-3.12 (Jaruvongvanich et al., 2017). This study is consistent with the study undertaken by Yang et al. stating that uric acid level is significantly related to the prevalence of mild and severe steatosis $(\mathrm{p}<0.01)$ (Yang et al., 2018). Zheng et al. reported that elevated uric acid level independently shows positive associations with the prevalence of Non-alcoholic fatty liver disease (NAFLD) and the severity of fatty liver (Zheng et al., 2017).

This study has some limitations, including the small number of samples, its nature as the cross-sectional study and the used diagnostic methods that could not show a relationship between the severity of NAFLD and uric acid levels. The exact role of uric acid in the pathophysiological mechanisms of NASH remains to be the thing that must be explored.

\section{Conclusion}

There is a correlation between uric acid levels and NASH cases in NAFLD (Non-Alcoholic Fatty Liver Disease) patients with and without obesity. A significant correlation was found between NASH and uric acid in obese results $(19.5 \%, p=0.000)$ and non obese results $(10.1 \%, p=0.000)$. In fact, NAFLD is also significantly associated with uric acid levels in female patients.

\section{Source of Funding}

This study was conducted by means of self-funding. There is no external funding sources for this study.

\section{Ethical Clearance}

The protocol of this study has been approved by the Health Research Ethics Commission of the Hasanuddin University Medical Faculty/Wahidin Sudirohusodo Hospital in accordance with the ethical recommendations from the Helsinki Declaration of 1975.

\section{Competing Interests Statement}

No relevant potential conflict of interest to be declared.

\section{References}

Ali, N., Miah, R., Hasan, M., Barman, Z., Mou, A. D., Hafsa, J. M., ... \& Islam, F. (2020). Association between serum uric acid and metabolic syndrome: a cross-sectional study in Bangladeshi adults. Scientific Reports, 10(1), 1-7. https://doi.org/10.1038/s41598-020-64884-7 
Ballestri, S., Nascimbeni, F., Baldelli, E., Marrazzo, A., Romagnoli, D., \& Lonardo, A. (2017). NAFLD as a sexual dimorphic disease: role of gender and reproductive status in the development and progression of nonalcoholic fatty liver disease and inherent cardiovascular risk. Advances in therapy, 34(6), 1291-1326. https://doi.org/10.1007/s12325-017-0556-1

Bellentani, S., Scaglioni, F., Marino, M., \& Bedogni, G. (2010). Epidemiology of non-alcoholic fatty liver disease. Digestive diseases, 28(1), 155-161. https://doi.org/10.1159/000282080

Benedict, M., \& Zhang, X. (2017). Non-alcoholic fatty liver disease: An expanded review. World journal of hepatology, 9(16), 715. https://doi.org/10.4254/wjh.v9.i16.715

Fabbrini, E., Sullivan, S., \& Klein, S. (2010). Obesity and nonalcoholic fatty liver disease: biochemical, metabolic, and clinical implications. Hepatology, 51(2), 679-689. https://doi.org/10.1002/hep.23280

Fang, Y. L., Chen, H., Wang, C. L., \& Liang, L. (2018). Pathogenesis of non-alcoholic fatty liver disease in children and adolescence: From "two hit theory" to "multiple hit model". World journal of gastroenterology, 24(27), 2974. https://doi.org/10.3748/wjg.v24.i27.2974

Hardy, O. T., Czech, M. P., \& Corvera, S. (2012). What causes the insulin resistance underlying obesity?. Current opinion in endocrinology, diabetes, and obesity, 19(2), 81. https://doi.org/10.1097/MED.0b013e3283514e13

Jaruvongvanich, V., Ahuja, W., Wirunsawanya, K., Wijarnpreecha, K., \& Ungprasert, P. (2017). Hyperuricemia is associated with nonalcoholic fatty liver disease activity score in patients with nonalcoholic fatty liver disease: A systematic review and meta-analysis. Eur J Gastroenterol Hepatol, 29(9), 1031-5. https://doi.org/10.1097/MEG.0000000000000931

Jaruvongvanich, V., Ahuja, W., Wirunsawanya, K., Wijarnpreecha, K., \& Ungprasert, P. (2017). Hyperuricemia is associated with nonalcoholic fatty liver disease activity score in patients with nonalcoholic fatty liver disease: a systematic review and meta-analysis. European Journal of Gastroenterology \& Hepatology, 29(9), 1031-1035. https://doi.org/10.1186/1758-5996-4-3

Lefere, S., \& Tacke, F. (2019). Macrophages in obesity and non-alcoholic fatty liver disease: Crosstalk with metabolism. JHEP reports, 1(1), 30-43. https://doi.org/10.1016/j.jhepr.2019.02.004

Park, J. W., Lee, J. H., Cho, H. J., Ha, Y. J., Kang, E. H., Shin, K., .. \& Lee, Y. J. (2018). Influence of androgen deprivation therapy on serum urate levels in patients with prostate cancer: A retrospective observational study. PloS one, 13(12), e0209049. https://doi.org/10.1371/journal.pone.0209049

Petta, S., Camma, C., Cabibi, D., Di Marco, V., \& Craxì, A. (2011). Hyperuricemia is associated with histological liver damage in patients with non-alcoholic fatty liver disease. Alimentary pharmacology \& therapeutics, 34(7), 757-766. https://doi.org/10.1111/j.1365-2036.2011.04788.x

Souza, M. R. D. A., Diniz, M. D. F. F. D. M., Medeiros-Filho, J. E. M. D., \& Araújo, M. S. T. D. (2012). Metabolic syndrome and risk factors for non-alcoholic fatty liver disease. Arquivos de gastroenterologia, 49(1), 89-96. https://doi.org/10.1590/S0004-28032012000100015

Wang, H., Zhang, H., Sun, L., \& Guo, W. (2018). Roles of hyperuricemia in metabolic syndrome and cardiac-kidney-vascular system diseases. American Journal of Translational Research, 10(9), 2749.

Wong, T., Wong, R. J., \& Gish, R. G. (2019). Diagnostic and treatment implications of nonalcoholic fatty liver disease and nonalcoholic steatohepatitis. Gastroenterology \& Hepatology, 15(2), 83.

Yang, H., Li, D., Song, X., Liu, F., Wang, X., Ma, Q., ... \& Li, X. (2018). Joint associations of serum uric acid and ALT with NAFLD in elderly men and women: a Chinese cross-sectional study. Journal of Translational Medicine, 16(1), 285. https://doi.org/10.1186/s12967-018-1657-6

Yang, C., Yang, S., Xu, W., Zhang, J., Fu, W., \& Feng, C. (2017). Association between the hyperuricemia and nonalcoholic fatty liver disease risk in a Chinese population: A retrospective cohort study. PLoS One, 12(5), e0177249. https://doi.org/10.1371/journal.pone.0177249

Younossi, Z., Anstee, Q. M., Marietti, M., Hardy, T., Henry, L., Eslam, M., ... \& Bugianesi, E. (2018). Global burden of NAFLD and NASH: trends, predictions, risk factors and prevention. Nature reviews Gastroenterology \& hepatology, 15(1), 11. https://doi.org/10.1038/nrgastro.2017.109

Yu, X. L., Shu, L., Shen, X. M., Zhang, X. Y., \& Zheng, P. F. (2017). Gender difference on the relationship between hyperuricemia and nonalcoholic fatty liver disease among Chinese: An observational study. Medicine, 96(39). http://dx.doi.org/10.1097/MD.0000000000008164 
Zhang, C., Song, H., Yang, L., Liu, Y., \& Ji, G. (2018). Does high level of uric acid lead to nonalcoholic fatty liver disease. GJ Dig. Dis, 4(3), 7-14.

Zheng, X., Gong, L., Luo, R., Chen, H., Peng, B., Ren, W., \& Wang, Y. (2017). Serum uric acid and non-alcoholic fatty liver disease in non-obesity Chinese adults. Lipids in Health and Disease, 16(1), 202. https://doi.org/10.1186/s12944-018-0712-x

\section{Copyrights}

Copyright for this article is retained by the author(s), with first publication rights granted to the journal.

This is an open-access article distributed under the terms and conditions of the Creative Commons Attribution license (http://creativecommons.org/licenses/by/4.0/). 